\title{
Políticas de saúde pública aplicadas à genética médica no Brasil
}

\author{
Joanna Góes Castro Meira \\ Angelina Xavier Acosta ${ }^{2}$
}

\begin{abstract}
Resumo
A participação dos defeitos congênitos na mortalidade infantil tornou-se mais significativa com o declínio das demais causas, por melhorias das condiçôes socioeconômicas, o que desperta maior interesse entre os profissionais de saúde pública. De acordo com a Organização Mundial de Saúde (OMS), 7,6 milhões de crianças nascem anualmente com um defeito genético grave. O Brasil apresenta-se heterogêneo em relação às taxas de mortalidade infantil, devido às disparidades socioeconômicas, e no alcance dos programas básicos de saúde. Este trabalho objetiva conhecer e descrever os programas de saúde pública, governamentais e não-governamentais, em vigência no Brasil e, em particular, no Estado da Bahia, relacionados à prevenção, manejo e tratamento das doenças genéticas. Dentre as açôes governamentais relacionadas à prevenção e monitorização das doenças genéticas, estão o Programa Nacional de Triagem Neonatal, o Programa de fortificação das farinhas com ácido fólico, a Implantação do Campo 34 na Declaração Nacional de Nascidos Vivos. Dentre as ações não-governamentais relacionadas à prevenção e monitorização das doenças genéticas, serão citados o Estudo Colaborativo Latino-americano de Malformaçōes Congênitas e o Sistema Nacional de Informação sobre Agentes Teratogênicos. Quanto às açōes relacionadas ao manejo e tratamento das doenças genéticas, existem o Programa de Osteogênese Imperfeita e o da Doença de Gaucher.
\end{abstract}

Palavras-chave: Saúde pública - Políticas - Brasil - Doenças genéticas; Defeitos congênitos - Mortalidade infantil.

\section{INTRODUÇÃO}

A maioria dos defeitos congênitos decorre de uma combinação de fatores genéticos e ambientais ${ }^{1,2,3}$. Segundo Kalter e Warkany, citados por Schüler-Faccini ${ }^{4}$, causas genéticas parecem ser responsáveis por 15 a $20 \%$ desses defeitos; fatores ambientais são reconhecidamente responsáveis por $7 \%$ dos casos; $20 \%$ são de etiologia multifatorial.
Porém, em mais de $50 \%$ dos casos, a causa permanece desconhecida.

Segundo dados da Organização Mundial de Saúde (OMS), 7,6 milhôes de crianças nascem a cada ano com um defeito ou desordem genética grave, sendo 95\% delas nos países em desenvolvimento, onde $80 \%$ da população global contribui significativamente para a mortalidade infantil. $^{2}$

\footnotetext{
${ }^{1}$ Médica Residente em Genética Médica no Hospital das Clínicas da USP Ribeirão Preto.

${ }^{2}$ Professora Dra Docente do Departamento de Pediatria da Faculdade de Medicina da Universidade Federal da Bahia.

Correspondência para / Correspondence to:

Lilia Maria de Azevedo Moreira.

Rua Campos Salles, 423, apt. 83 - Centro.

14015-110 Ribeirão Preto - SP - Brasil.

Tel.: (16) 8128-5454; 3234-3712.

E-mail: joannagoes@yahoo.com.br.
} 
Com o declínio da mortalidade infantil, devido às melhorias das condições socioeconômicas, a contribuição de doenças genéticas para esse índice tornou-se proporcionalmente mais alta, devido também à sua maior dificuldade de prevenção, o que despertando maior interesse entre os profissionais de saúde pública acerca do assunto. ${ }^{5}$

Observa-se a alteração do perfil das causas da mortalidade infantil, no Brasil, para crianças menores de um ano. Em 1980, as causas perinatais eram as principais causas de mortalidade infantil, e as anomalias congênitas ocupavam a quinta posição, com 5\% do total. Em 2000, houve grande redução proporcional dos óbitos por causas infecciosas e respiratórias, e as anomalias congênitas passaram a ocupar a segunda posição, passando para $10 \% .^{6}$

Segundo dados do DATASUS/SINASC, no ano de 2004, na Bahia, foram verificados um total de 235.337 nascimentos, sendo que em 1.114 $(0,47 \%)$ foram identificados com anomalias ao nascimento. $\mathrm{Na}$ região metropolitana de Salvador, no mesmo período, houve 51.545 nascimentos, sendo 544 (1,05\%) classificados com presença de anomalias ${ }^{7}$. A prevenção primária de anomalias congênitas aparece como um problema emergente em nosso meio, devendo ser investigado.

Os dados aqui disponibilizados de maneira organizada e revisada podem contribuir para o conhecimento dos programas que vêm sendo implantados pelo setor público no Brasil, no âmbito da genética médica, tendo em vista o pouco conhecimento e divulgação dessas políticas públicas já existentes em nosso meio.

Este trabalho tem como objetivo caracterizar o perfil epidemiológico das patologias no âmbito da genética médica e conhecer e descrever os programas de saúde pública em vigência no Brasil e, em particular, no Estado da Bahia, relacionados à prevenção, manejo e tratamento das doenças genéticas, identificando os programas governamentais e não-governamentais relacionados.

\section{METODOLOGIA}

O estudo constitui uma revisão bibliográfica de caráter descritivo. A coleta de dados foi realizada no acervo de livros-texto e revistas especializadas em bibliotecas e livrostexto de acervo pessoal.

Foram feitas também pesquisas nas bases de dados SCIELO, MEDLINE e LILACS, no período de 1996-2006, e em websites específicos, como o site do Ministério da Saúde, entre outros, para estudo da legislação.

\section{DESENVOLVIMENTO}

\section{Ações governamentais relacionadas à prevenção e monitorização das doenças genéticas}

\section{Programa Nacional de Triagem Neonatal (PNTN)}

O PNTN foi criado através da Portaria GM/MS no 822 , de 6 de junho de $2001^{8}$, com os seguintes objetivos específicos: ampliação da cobertura, visando a $100 \%$ dos nascidos vivos, busca ativa dos pacientes triados, sua confirmação diagnóstica, acompanhamento e tratamento adequados dos pacientes identificados.

As patologias selecionadas para fazer parte do programa teriam como características: ausência de manifestações clínicas precoces, possibilidade de detecção precoce por meio de testes confiáveis, serem amenizáveis mediante tratamento, serem logisticamente administráveis, e terem uma relação custobenefício economicamente viável. As patologias incluídas nos programas são a fenilcetonúria, o hipotireoidismo congênito, as hemoglobinopatias e a fibrose cística. ${ }^{6,9,10}$

A APAE Salvador é responsável por toda a cobertura dos "Testes do Pezinho" realizados pelo SUS na Bahia, sendo o Serviço de Referência em Triagem Neonatal (SRTN) do estado, o qual se encontra na fase 2 do PNTN ${ }^{11}$, ou seja, realiza testes para fenilcetonúria, hipotireoidismo congênito e hemoglobinopatias. Almeida e colaboradores $^{11}$ avaliaram o perfil do PNTN baiano no ano de 2003, obtendo os seguintes dados: cobertura de $94,5 \%$ dos municípios; cobertura de 72,51\% dos neonatos; incidência de 1:22.000 para fenilcetonúria, 1:4.000 para o hipotireoidismo congênito e 1:650 para as 
hemoglobinopatias. O objetivo de alcançar $100 \%$ de cobertura dos RN do país pode ser dificultado por problemas socioeconômicos e culturais e subestimação dos dados, devido à realização de testes na rede privada. Segundo dados de 2005 disponibilizados recentemente pela equipe da APAE Salvador, a cobertura populacional estimada nesse ano pelo Teste do Pezinho foi de 79\%; a cobertura dos municípios foi de $97,8 \%$, e as frequências observadas para as patologias envolvidas no PNTN mantiveramse constantes.*

\section{$\underline{\text { Fenilcetonúria (PKU) }}$}

A PKU é a patologia mais prevalente entre os erros inatos no metabolismo de aminoácidos, resultando em incapacidade de metabolismo do aminoácido fenilalanina (Phe) em tirosina $(\mathrm{Tyr})^{12,13}$. A PKU ocorre em todos os grupos étnicos e, devido à grande variabilidade genética, a incidência em recém-nascidos ( $R N$ ) pode variar de 1:2.600 até $1: 26.000$, sendo a média de $1: 10.000 .^{13}$

Através da Portaria SAS no 847 de 06 de Novembro de $2002{ }^{14}$, foi aprovado um protocolo que contém o perfil da PKU e a regulação da dispensação da fórmula de aminoácidos isenta de fenilalanina. A restrição dietética de Phe, iniciada antes de um mês de idade, é altamente efetiva em alterar o curso da doença e o dano neurológico. ${ }^{15}$

Foram analisados 51 pacientes com diagnóstico de hiperfenilalaninemia acompanhados no SRTN/BA. Cerca de 78,4\% dos pacientes teve seu diagnóstico através da triagem neonatal, tendo, portanto, tratamento precoce. Porém há obstáculos, como falta de informação da população leiga e dos profissionais de saúde sobre a importância da precocidade na coleta do teste e posterior tratamento, o que pode comprometer o prognóstico desses pacientes. ${ }^{16}$

\section{Hipotireoidismo Congênito (HC)}

$\mathrm{O} \mathrm{HC}$ ocorre em crianças que nascem com a incapacidade de produzir quantidades normais de hormônio da tireóide, o qual é essencial para o crescimento físico e neurológico normal. ${ }^{17}$

A maior parte dos RN é geralmente assintomática até o terceiro mês de vida, mesmo com o dano cerebral já ocorrendo progressivamente $^{18}$. O tratamento deve ser iniciado preferencialmente com até um mês de vida, com administração oral de levotiroxina, sendo considerado tardio após esse período. ${ }^{15}$

\section{$\underline{\text { Hemoglobinopatias }}$}

As hemoglobinopatias podem ser anomalias estruturais, em que a molécula de hemoglobina $(\mathrm{Hb})$ é alterada, e as talassemias, em que a estrutura $\mathrm{Hb}$ é normal, embora sua quantidade seja reduzida ${ }^{12}$. A OMS estima que, da população mundial, aproximadamente 5\% possuem mutações de genes envolvidos na síntese da Hb. ${ }^{15}$

Segundo Silla, a anemia falciforme (AF) é a hemoglobinopatia (anomalia estrutural) mais frequente no Brasil, atingindo cerca de 0,1 a $0,3 \%$ da população de negros. No país, a alta taxa de miscigenação difundiu a doença em praticamente todos os grupos populacionais. ${ }^{10}$ Em relação ao tratamento da AF, é fundamental a prevenção de infecçõos com imunização para hepatite $\mathrm{B}$, anti-hemófilo, anti-meningocócica e anti-pneumocócica; profilaxia com penicilina $\mathrm{V}$ oral a partir de 2 a 3 meses até os 5 anos de idade; o ácido fólico deve ser utilizado como em qualquer outro estado hemolítico crônico. As crises álgicas vaso-oclusivas devem ser tratadas com hidratação parenteral, analgesia com opiáceos, administração de oxigênio e tratamento da causa precipitante, se presente, no caso das infecçôes ${ }^{17}$. A Portaria no 872 de 12 de Novembro de $2002{ }^{19}$ da Secretaria de Assistência à Saúde, estabeleceu o Protocolo Clínico e Diretrizes Terapêuticas para o tratamento da Doença Falciforme, disponibilizando medicamento preconizado para o tratamento da doença, no caso a hidroxiuréia, que é recomendada para pacientes com história de internamentos e crises álgicas vaso-oclusivas frequentes.

\footnotetext{
* Dados fornecidos por Maria Inês M. M. Fontes do Centro de
} Diagnóstico e Pesquisa do Serviço de Referência em Triagem 
O diagnóstico neonatal e o tratamento precoce dessas hemoglobinopatias aumentam significativamente a sobrevivência e a qualidade de vida dos seus portadores, diminuindo as suas sequelas e atenuando as suas complicações clínicas. ${ }^{20}$

\section{Fibrose Cística (FC)}

A FC ou mucoviscidose é uma patologia caracterizada por uma disfunção no transporte epitelial de íons cloreto, causada por mutações no gene que codifica uma proteína reguladora da condutância transmembrana da $\mathrm{FC}^{10,12,15}$. Essas alterações podem afetar diversos órgãos que secretam muco, inclusive o aparelho respiratório, o pâncreas, o sistema biliar, a genitália masculina, $\mathrm{o}$ intestino e as glândulas sudoríparas. ${ }^{21}$

Nos Estados Unidos, a FC apresenta uma frequência de $1: 2.000$ a 1:4.000 neonatos caucasianos, prevalência de 1:17.000 entre os afroamericanos e muito rara entre os asiáticos, com prevalência de 1:90.000. Cerca de 30.000 americanos são acometidos por essa doença ${ }^{12}$. A FC tem elevada prevalência entre populações de origem européia, e sua frequência, na população do sul do Brasil, é de aproximadamente 1:2500 nativivos. ${ }^{10}$

Em 18 de julho de 2001, foi aprovada a Portaria no $263^{22}$ da Secretaria de Assistência à Saúde, que determinou o Protocolo Clínico e Diretrizes Terapêuticas com disponibilização de enzimas pancreáticas para o tratamento da FC. A descoberta prematura da doença permite que os indivíduos portadores recebam orientação, acompanhamento médico e tratamento quando necessário. ${ }^{17}$

Programa de fortificação das farinhas com ácido fólico

Os Defeitos do Tubo Neural (DTN), durante o desenvolvimento embrionário, constituem um grupo das malformações das mais pesquisadas, devido à sua alta prevalência e mortalidade ${ }^{15}$. Trata-se de alterações no fechamento do tubo neural, o que leva à anencefalia, encefalocele e à espinha bífida. ${ }^{12}$

Recentemente, o uso periconcepcional de folato e multivitamínicos foi descoberto como método de prevenção de ocorrência e recorrência de $\mathrm{DTN}^{12}$. Uma combinação de ácido fólico preventivo e triagem de á-feto-proteína no soro materno beneficiarão a saúde pública, reduzindo drasticamente a incidência de DTN. ${ }^{15}$

Em 13 dezembro de 2002, foi regulamentada, através da Resolução no $344{ }^{23}$, pela Agência Nacional de Vigilância Sanitária (ANVISA), a fortificação da farinha de trigo e de milho com ferro e ácido fólico, seguindo as recomendações da OMS e da Organização Panamericana da Saúde (OPAS). Acredita-se que 50 a $70 \%$ dos DTN podem ser evitados simplesmente com a suplementação dietética do ácido fólico. ${ }^{12}$

Foi analisada a prevalência de DTN em hospitais da rede do ECLAMC, no período de 1999 a 2001, após a fortificação das farinhas com ácido fólico em países da América do Sul. Verificou-se que o Chile vem adicionando doses suficientes para a efetiva prevenção, apresentando redução de $31 \%$ na taxa de prevalência de DTN. ${ }^{24}$

Mesmo após mais de dez anos de estudos que demonstram que o ácido fólico previne DTN, apenas uma minoria das mulheres fazem uso dos suplementos com essa substância no período periconcepcional e apenas poucos países responderam recentemente a essa introdução da suplementação, muitos deles com doses insuficientes. $^{24}$

Implantação do Campo 34 na Declaração Nacional de Nascidos Vivos

A partir de janeiro de 2000, houve a introdução de um novo campo de registro obrigatório na Declaração Nacional de Nascidos Vivos (DNV), o Campo 34. É nesse campo que são registradas a ausência ou presença de malformações ao nascimento, sua descrição e classificação de acordo com o CID-10, permitindo que equipes de informação da saúde, locadas nas Secretarias Municipais de Saúde, registrem sistematicamente as anomalias congênitas. ${ }^{4,6}$

Dados como esses são raros na maioria dos países, sendo de grande valia para a monitorização de um quadro epidemiológico 
acerca dos defeitos congênitos no país ${ }^{6}$. Infelizmente, ainda existe um sub-registro de anomalias congênitas, devido ao não preenchimento ou o mau preenchimento do Campo 34, sendo indispensáveis ações de educação dos profissionais de saúde sobre seu correto preenchimento, destacando a importância de sua implementação efetiva, para permitir o conhecimento da realidade nacional relacionada aos defeitos congênitos e, consequentemente, uma maior programação de ações de saúde para prevenção e manejo das doenças genéticas.

Ações não-governamentais relacionadas à prevenção e monitorização das doenças genéticas

ECLAMC - Estudo Colaborativo Latino Americano de Malformaçôes Congênitas

O ECLAMC é uma entidade nãogovernamental, criada em 1967, que funciona como um programa de investigação dos defeitos congênitos e suas causas, sendo reconhecido pela OMS como um centro colaborativo de prevenção de defeitos congênitos ${ }^{25}$. Conta com 70 hospitais distribuídos em 10 países da América do Sul e Caribe (no Brasil são cerca de 10 hospitais participantes $)^{4}$. A Bahia é um estado participante do ECLAMC desde novembro de 2002, quando o programa foi implantado na Maternidade Climério de Oliveira, ligada à Universidade Federal da Bahia.

O ECLAMC objetiva investigar os fatores de risco e as causas dos defeitos congênitos, através da metodologia de casocontrole e, como mais da metade das anomalias tem causa desconhecida, o principal objetivo estratégico do ECLAMC é a prevenção por meio da investigação e vigilância permanente. Atua como um sistema de vigilância epidemiológica, observando sistematicamente as flutuações nas frequências dos diferentes defeitos congênitos e, frente à suspeita de epidemia para um tipo de anomalia, em determinados momento e em determinadas áreas, mobiliza-se para tentar identificar suaa causa. ${ }^{4,6,25}$
SIAT - Sistema Nacional de Informação sobre Agentes Teratogênicos

Teratógenos são definidos como qualquer agente externo ao genoma do feto que induz defeitos estruturais, deficiência de crescimento e (ou) alterações funcionais no período de desenvolvimento pré-natal. ${ }^{12}$

Existem inúmeros agentes teratogênicos e novas associações são descobertas a cada dia. Logo, é de extrema importância a criação de centros de informação sobre teratógenos e suas consequências para profissionais de saúde e a população em geral.

Em 1990, foi criado o Sistema Nacional de Informação sobre Agentes Teratogênicos (SIAT), inicialmente implantado na América Latina. No Brasil, foi criado na cidade de Porto Alegre pelo Serviço de Genética Médica do Hospital das Clínicas de Porto Alegre, sendo vinculado ao ECLAMC ${ }^{25}$. Atualmente, existem outros SIAT operando no Brasil, nas cidades de Salvador, Rio de Janeiro, São Paulo, Campinas, Juazeiro e Fortaleza.

O SIAT consiste em um serviço telefônico, ou via internet ou fax, sem custos para o usuário, cujo objetivo é disponibilizar informaçôes sobre riscos ao concepto relacionados à exposição de gestantes a agentes químicos, físicos e biológicos, sendo destinados a gestantes, médicos ou pesquisadores $^{6}$. Em Salvador (BA), o SIAT funciona no Hospital Universitário Professor Edgard Santos (HUPES) desde 2001, no Serviço de Genética Médica, com a participação de médicos e estudantes de medicina treinados e supervisionados.

\section{Ações governamentais relacionadas ao manejo e tratamento das doenças genéticas}

\section{Programa de Osteogênese Imperfeita (OI)}

A OI é uma doença genética que engloba um grupo de mutaçôes do colágeno tipo I, caracterizada por fragilidade óssea e osteopenia, cujo quadro clínico é de múltiplas fraturas ósseas, deformidade óssea e limitação funcional ${ }^{6,15}$. O MS estima que, no Brasil, existam pelo menos 12.000 portadores de OI. ${ }^{26}$ 
Medidas cirúrgicas e médicas, em geral, contribuem para o tratamento dessa patologia. Entretanto, efeitos benéficos estão sendo atribuídos aos bifosfonatos, que reduzem a reabsorção óssea, aumentando a densidade do osso e, consequentemente, reduzindo o número de fraturas e melhorando a qualidade de vida do paciente acometido. Em 19 de dezembro de 2001, foi instituído, através da Portaria n. ${ }^{\circ}$ 2305/GM, o Protocolo de Indicação de Tratamento Clínico da OI com pamidronato dissódico no âmbito do SUS. ${ }^{27}$

No Brasil, segundo a Portaria no 2.305 27, existem 11 centros de referências em OI (CROI) reconhecidos pelo MS para o diagnóstico, acompanhamento e tratamento da OI espalhados nos estados do CE, PE, ES, RJ, SP, PR, RS e Distrito Federal. Na Bahia, o estabelecimento do Hospital Universitário Professor Edgard Santos da UFBA como centro de referência para OI encontra-se em andamento.

\section{Programa da Doença de Gaucher (DG)}

A DG constitui uma patologia metabólica, do grupo das glicoesfingolipidoses, que afeta o metabolismo dos lipídeos ${ }^{6,21}$. Há cerca de 20.000 pacientes diagnosticados com DG ao redor do mundo. ${ }^{28}$

Em 08 de julho de 2002, foi publicada a Portaria no 449 da Secretaria de Assistência à Saúde do MS, aprovando o protocolo clínico e diretrizes terapêuticas para a DG, custeando, assim, o tratamento com Imiglucerase. ${ }^{29}$

No Brasil, há 510 casos diagnosticados e 480 em tratamento, porém não há estudos epidemiológicos. Na Bahia, são 18 casos acompanhados no HEMOBA.

Outras doenças metabólicas já possuem tratamentos de reposição enzimática aprovados pelo FDA (Food and Drug Administration) dos Estados Unidos, que, entretanto, não estão incluídas nos programas brasileiros como a Mucopolissacaridose tipo I, II e VI, a Doença de Fabry e a Doença de Pompe ${ }^{30}$. Em muitos casos, as Organizações Não-Governamentais

* Dados fornecidos por Dra. Elisa Sobreira do serviço de Hemato-
(ONG) e associações de pais e familares de portadores de doenças genéticas vêm mobilizando o governo através de ações judiciais para garantir o direito à medicação para essas patologias não regulamentadas pelo Estado, porém com tratamentos aprovados pela FDA.

\section{CONSIDERAÇÕES FINAIS}

Em países em desenvolvimento, ainda se questiona se os programas para prevenção, manejo e tratamento das doenças genéticas devam ser implantados, já que as doenças são mais raras e os recursos disponíveis para a saúde são escassos. Entretanto, as doenças genéticas apresentam alta morbimortalidade e necessitam de tratamento contínuo e oneroso. Além disso, essas patologias são freqüentemente subdiagnosticadas, perdendo oportunidades para prevenção e orientações antecipadas, o que impõe altos custos aos pacientes, seus familiares e ao sistema de saúde em geral. ${ }^{2}$

As ONGs e associações têm o importante papel de mobilizar a opinião pública e conseguir o apoio da população para melhorar o panorama atual, divulgando as patologias e exigindo melhoras nas condições de diagnóstico, acompanhamento e tratamento dos pacientes, podendo complementar o trabalho do Estado.

O grupo de trabalho para implementação da Política Nacional de Atenção à Saúde em Genética Clínica (PNASGC) foi instituído em 2004 pelo MS, determinando, no SUS, que as secretarias de saúde estaduais e municipais sejam responsáveis por assegurar as condições necessárias para implementação de redes de atendimento em Genética Clínica. O ponto principal dessa política é a Atenção Básica, priorizando-se a prevenção, com a finalidade de identificar indivíduos acometidos por doenças genéticas e também seus familiares para, posteriormente, receberem acompanhamento na Atenção Especializada, incluindo o aconselhamento genético. Diante da aprovação dessa política de saúde, a expectativa é de melhora do quadro atual, para que pacientes e familiares tenham suas necessidades atendidas. ${ }^{26}$ 


\title{
Public health programs applied to medical genetics in Brazi
}

\begin{abstract}
The participation of genetic defects in child death rate became more significant after the decline of the others death causes, due to the improvement in the social-economic conditions, getting the focus of health professionals. According to World Health Organization (WHO), every year, 7,6 million of children are born with a serious genetic defect. Brazil is heterogeneous in its child death rate because of the high socialeconomic differences and because of basic health programs impacts. This study aims to know and to describe the governmental and non-governmental public health programs in Brazil nowadays, more precisely in Bahia state, related to prevention and treatment of genetic diseases. Among the governmental actions related to prevention and monitoring of the genetic disease is the "Programa Nacional de Triagem Neonatal", the program for folic acid flour enrichment and the implementation of "Campo 34" in the "Declaração Nacional de Nascidos Vivos". Among the non-governmental actions related to the prevention and monitoring of the genetic diseases, will be discussed the "Estudo Colaborativo Latino Americano de Malformaçoes Congênitas" and the "Sistema Nacional de Informaçôes sobre Agentes Teratogênicos". About the actions related to treatment of the genetic diseases the programs: "Programa de Osteogênese Imperfeita" and the "Doença de Gaucher" will be discussed.
\end{abstract}

Keywords: Public health programs-Brazil - Genetic diseases; Congenital defects-Child death rate.

\section{REFERÊNCIAS}

1 LEITE, J.C.L. et al. Tópicos em defeitos congênitos. Porto Alegre: Ed. da Universidade, 2002.

2 MARQUES-DE-FARIA, A.P. et al. Clinical genetics in developing countries: the case of Brazil. Community Genet., Basel, v.7, n.2/3, p.95-105, 2004.

3 RASMUSSEN, S.A.; MOORE, C.A. Public health approach to birth defects, developmental disabilities, and genetic conditions. Am. J. Med. Genet. C Semin. Med. Genet., v.125C, n.1, p.1-3, 2004.

4 SCHÜLER-FACCINI, L. et al. Avaliação de teratógenos potenciais na população brasileira. Ci. Saúde Coletiva, Rio de Janeiro, v.7, n.1, p.65-71, 2002.

5 MENEZES, A.M.B. et al. Mortalidade infantil em duas coortes de base populacional no Sul do Brasil: tendências e diferenciais. Cad. Saúde Pública, Rio de Janeiro, v.12, n.1, p.79-86, 1996.

6 HOROVITZ, D.D.G.; LLERENA JR., J.C.; MATTOS, R.A. Atenção aos defeitos congênitos no Brasil: panorama atual. Cad. Saúde Pública, Rio de Janeiro, v.21, n.4, p.1055-1064, jul./ago. 2005.
7 BRASIL. Ministério da Saúde. Departamento de Informática do SUS. Estatísticas vitais: mortalidade e nascidos vivos. Brasília, DF, 2008. Disponível em: <http://w3.datasus.gov.br/ datasus/datasus.php?are../site/ in f s a u d e.ph p \& V O b j = h t t p : / / tabnet.datasus.gov.br/cgi/deftohtm.exe?sinasc/ cnv/nv>. Acesso em: 15 maio 2007.

8 BRASIL. Ministério da Saúde. Portaria n.822, de 6 de junho de 2001. Institui, no âmbito do Sistema Único de Saúde, o Programa Nacional de Triagem Neonatal/PNTN. Brasília, DF, 2001. Disponível em: <http://dtr2001.saude.gov.br/ sas / PORTARIAS/Port2001/GM / GM822.htm>. Acesso em: 8 ago. 2006.

9 BRUNONI, D. Aconselhamento genético. Ci. Saúde Coletiva, Rio de Janeiro, v.7, n.1, p.101-107, 2002.

10 SOUZA, C.F.M.; SCHWARTZ, I.V.; GIUGLIANI, R. Triagem neonatal de distúrbios metabólicos. Ci. Saúde Coletiva, Rio de Janeiro, v.7, n.1, p.129-137, 2002.

11 ALMEIDA, A.M. et al. Avaliação do Programa de Triagem Neonatal na Bahia no ano de 2003. R. Bras. Saúde Mater. Infant., Recife, v.6, n.1, p.85-91, jan./mar. 2006. 
12 JORDE, L.B. et al. Genética médica. Tradução por Paulo Armando Motta. 2.ed. Rio de Janeiro: Guanabara Koogan, 2000.

13 MIRA, N.V.M. de; MARQUEZ, U.M.L. Importância do diagnóstico e tratamento da fenilcetonúria. R. Saúde Públ., São Paulo, v.34, n.1, p.86-96, fev. 2000. Disponível em: <http:/

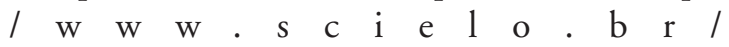
scielo.php?script $=$ sci_arttext $\&$ pid $=S 0034-$ $89102000000100016 \& \operatorname{lng}=\mathrm{en} \& \mathrm{nrm}=$ iso $>$. Acesso em: 16 ago. 2006.

14 BRASIL. Ministério da Saúde. Secretaria de Assistência à Saúde. Portaria n.847, de 6 de novembro de 2002. Aprova o protocolo clínico e diretrizes terapêuticas - Fenilcetonúria - Fórmula de aminoácidos isenta de fenilalanina. Brasília, DF, 2002. Disponível em: <http://dtr2001.saude.gov.br/ sas/PORTARIAS/PORT2002/PT-847.htm>. Acesso em: 8 ago. 2006.

15 NUSSBAUM, R.L.; MCINNES, R.R.; WILLARD, H.F. Thompson \& Thompson genética médica. Tradução por Paulo Armando Motta. 6.ed. Rio de Janeiro: Guanabara Koogan, 2002.

16 AMORIM, T. et al. Aspectos clínicos da fenilcetonúria em serviço de referência em triagem neonatal da Bahia. R. Bras. Saúde Mater. Infant., Recife, v.5, n.4, p.457-462, out./dez. 2005.

17 GOLDMAN, L.; BENNETT, J.C. Cecil Tratado de medicina interna. Tradução Amaury José da Cruz Junior. 21ed. Rio de Janeiro: Guanabara Koogan, 2001.

18 RAMALHO, R.J.R.; VALIDO, D.P.; AGUIAR-OLIVEIRA, M.H. Avaliação do programa de triagem para o hipotireoidismo congênito no Estado de Sergipe. Arq. Bras. Endocrinol. Metabol., São Paulo, v.44, n.2, p.157-161, abr. 2000.

19 BRASIL. Ministério da Saúde. Secretaria de Assistência à Saúde. Portaria n.872, de 12 de novembro de 2002. Aprova o protocolo clínico e diretrizes terapêuticas - Doença falciforme Hidroxiuréia. Brasília, DF, 2002. Disponível em: < http://dtr2001.saude.gov.br/sas/ PORTARIAS/PORT2002/PT-872.htm >. Acesso em: 1 set. 2006.
20 RAMALHO, R.J.R.; MAGNA, L.A.; PAIVA-E-SILVA, R.B. A Portaria n.822/01 do Ministério da Saúde e as peculiaridades das hemoglobinopatias em saúde pública no Brasil. Cad. Saúde Pública, Rio de Janeiro, v.19, n.4, p.1195-1199, jul./ago. 2003.

21 LEWIS, R. Genética humana: conceitos e aplicaçôes. Tradução por Paulo Armando Motta. 5.ed. Rio de Janeiro: Guanabara Koogan, 2004.

22 BRASIL. Ministério da Saúde. Secretaria de Assistência à Saúde. Portaria n.263, de 18 de julho de 2001. Aprova o protocolo clínico e diretrizes terapêuticas - Fibrose cística - Enzimas pancreáticas. Brasília, DF, 2001. Disponível em: < http://dtr2001.saude.gov.br/sas/PORTARIAS/ Port2001/PT-263.htm>. Acesso em: 5 set. 2006.

23 AGÊNCIA NACIONAL DE VIGILÂNCIA SANITÁRIA (Brasil). Resolução RDC n.344, de 13 de dezembro de 2002. Aprova o regulamento técnico para a fortificação das farinhas de trigo e das farinhas de milho com ferro e ácido fólico. Diário Oficial [da] República Federativa do Brasil, Poder Executivo, Brasília, DF, 18 dez. 2002. Disponível em: < http://e-legis.anvisa.gov.br/ leisref/public/showAct.php?id=1679>. Acesso em: 8 ago. 2006.

24 CASTILLA, E.E. et al. Preliminary data on changes in neural tube defect prevalence rates after folic acid fortification in South America. Am. J. Med. Genet. A, Hoboken, v.123, p.123-128, 2003.

25 CASTILLA, E.E; ORIOLI, I.M. ECLAMC: The Latin-American Collaborative Study of Congenital Malformations. Community Genet., Basel, v.7, p.76-94, 2004.

26 BRASIL. Ministério da Saúde. Portal da Saúde. Disponível em: <http://portal.saude.gov.br/saude/ >. Acesso em: 1 ago. 2006.

27 BRASIL. Ministério da Saúde. Portaria n.2.305, de 19 de dezembro de 2001. Aprova o Protocolo de Indicação de Tratamento Clínico da osteogenesis imperfecta com pamidronato dissódico no âmbito do Sistema Único de Saúde. Brasília, DF, 2001. Disponível em: <http://sna.saude.gov.br/legisla/ legisla/informes/GM_P2.305_01informes.doc>. Acesso em: 20 ago. 2006. 
28 Mc Cormack, P.L.; GOA, K.L. Miglustat. Drugs, Auckland, v.63, n.22, p.2427-2434, 2003.

29 BRASIL. Ministério da Saúde. Secretaria de Assistência à Saúde. Portaria n.449, de 8 de julho de 2002. Aprova o protocolo clínico e diretrizes terapêuticas - Doença de Gaucher Imiglucerase. Diário Oficial [da] República Federativa do Brasil, Brasília, DF, 9 jul. 2002.
Disponível em: <http://sna.saude.gov.br/legisla/ $\mathrm{l}$ e g i s l a / i n f o r m e s / SAS_P449_02informes.doc>Acesso em: 8 ago. 2006.

30 OLIVEIRA, M.C.L.A. et al. Aspectos clínicos e nutricionais da doença de Gaucher: estudo prospectivo de 13 crianças em um único centro. J. Pediatr. (Rio J.), Porto Alegre, v.78, n.6, p.517-522, nov./dez. 2002.

Recebido em / Received: 17/09/2008 Aceito em / Accepted: 30/04/2009 